\title{
Modeling the source of GW150914 with targeted numerical-relativity simulations
}

\author{
Geoffrey Lovelace, ${ }^{1}$ Carlos O. Lousto, ${ }^{2}$ James Healy ${ }^{2}$ Mark A. Scheel,${ }^{3}$ Alyssa Garcia,${ }^{1}$ \\ Richard O'Shaughnessy, ${ }^{2}$ Michael Boyle, ${ }^{4}$ Manuela Campanelli, ${ }^{2}$ Daniel A. Hemberger, ${ }^{3}$ \\ Lawrence E. Kidder, ${ }^{4}$ Harald P. Pfeiffer, ${ }^{5}$ Béla Szilágyi, ${ }^{3,}{ }^{3}$ Saul A. Teukolsky, ${ }^{4}$ and Yosef Zlochower ${ }^{2}$ \\ ${ }^{1}$ Gravitational Wave Physics and Astronomy Center, California State University Fullerton, Fullerton, California 92834, USA \\ ${ }^{2}$ Center for Computational Relativity and Gravitation, School of Mathematical Sciences, \\ Rochester Institute of Technology, 85 Lomb Memorial Drive, Rochester, New York, 14623, USA \\ ${ }^{3}$ Theoretical Astrophysics 350-17, California Institute of Technology, Pasadena, CA 91125, USA \\ ${ }^{4}$ Center for Astrophysics and Planetary Science, Cornell University, Ithaca, New York 14853, USA \\ ${ }^{5}$ Canadian Institute for Theoretical Astrophysics, 60 St. George Street, University of Toronto, Toronto, ON M5S 3H8, Canada \\ ${ }^{6}$ Caltech JPL, Pasadena, California 91109, USA
}

(Dated: July 20, 2016)

\begin{abstract}
In fall of 2015, the two LIGO detectors measured the gravitational wave signal GW150914, which originated from a pair of merging black holes [1]. In the final 0.2 seconds (about 8 gravitational-wave cycles) before the amplitude reached its maximum, the observed signal swept up in amplitude and frequency, from $35 \mathrm{~Hz}$ to $150 \mathrm{~Hz}$. The theoretical gravitational-wave signal for merging black holes, as predicted by general relativity, can be computed only by full numerical relativity, because analytic approximations fail near the time of merger. Moreover, the nearly-equal masses, moderate spins, and small number of orbits of GW150914 are especially straightforward and efficient to simulate with modern numerical-relativity codes. In this paper, we report the modeling of GW150914 with numerical-relativity simulations, using black-hole masses and spins consistent with those inferred from LIGO's measurement [2]. In particular, we employ two independent numerical-relativity codes that use completely different analytical and numerical methods to model the same merging black holes and to compute the emitted gravitational waveform; we find excellent agreement between the waveforms produced by the two independent codes. These results demonstrate the validity, impact, and potential of current and future studies using rapid-response, targeted numerical-relativity simulations for better understanding gravitational-wave observations.
\end{abstract}

PACS numbers: 04.25.D-,04.25.dg,04.30.-w,04.30.Db

\section{INTRODUCTION}

On September 14, 2015, the Advanced Laser Interferometer Gravitational-Wave Observatory (Advanced LIGO) directly measured gravitational waves for the first time [1], giving birth to a new era of astronomy. The waves were emitted by a pair of black holes with masses $36_{-4}^{+5} M_{\odot}$ and $29_{-4}^{+4} M_{\odot}[1,2]$ that orbited each other, collided, and merged about 1.3 billion years ago. The gravitational wave signal from this event, named GW150914, is consistent with general relativity [3]. LIGO has already observed a second gravitational-wave signal from merging black holes (called GW151226) [4] and a third possible signal (called LVT151012) [5]; many more such observations are expected soon. Extrapolating from the observations of GW150914 and GW151226 and including LVT151012, Advanced LIGO is expected to observe between roughly 5 to tens of gravitational-wave signals from merging black holes during its next six-month observing run $(\mathrm{O} 2)$ starting in 2016 [5].

The GW150914 observation included 8 gravitationalwave cycles, covering the late inspiral, merger, and ringdown phases of the binary (cf. Fig. 2 of Ref. [1]); this late phase of a binary-black-hole (BBH) merger can be described accurately only by directly solving the full equations of general relativity. To extract and validate robust conclusions about the astrophysical and fundamental sig- nificance of these events $[1-3,6]$, correct and complete solutions to Einstein's equations will be critical, and can be obtained only through direct numerical simulation.

The first attempts to solve the general relativity field equations numerically date to the 1960s, when Hahn and Lindquist [7] performed the first studies. Smarr followed these early efforts with some success in the 1970s [8, 9]. The field matured in the 1990s, when a large collaboration of research groups worked together toward solving the "Grand Challenge" of evolving BBHs [10]. The crucial final breakthrough came in 2005, when three groups [11-13] devised two completely independent techniques to numerically solve the $\mathrm{BBH}$ problem. The first solution [11] handled the spacetime singularity by excising the regions interior to the black hole horizons, while the second technique $[12,13]$, dubbed the "moving punctures approach," used singularity avoiding slices of the black hole spacetimes.

Since then, through considerable effort by many research groups (reviewed, e.g., in Refs [14-18]), each of these techniques have matured, yielding two distinct, independent approaches to modeling BBHs with numerical relativity. Important analytic and numerical developments [19-21] have improved the accuracy and efficiency of the codes implementing each technique. Each technique has enabled numerical relativists to begin constructing catalogs of $\mathrm{BBHs}$ and associated gravitational waveforms [20, 22-27]. A BBH can be characterized 
by seven intrinsic parameters: the ratio $q$ of the holes' masses and the spin-angular-momentum vectors $S_{1}^{i}$ and $S_{2}^{i}$ of each hole; as a result, these catalogs must include many $\mathrm{BBH}$ simulations to span the parameter space of $\mathrm{BBH}$ events that LIGO could observe. Note that early work has already used numerical-relativity waveforms for detection and parameter estimation in LIGO, for the injection of waveforms [22, 28-30], into LIGO noise, and for establishing common error measures to establish standard of required waveform accuracy [23].

In this paper, we present a detailed comparison of the targeted numerical $\mathrm{BBH}$ simulations that modeled GW150914 in Figs. 1-2 of Ref. [1], provided by two codes: SpEC and LazEv. We chose the parameters (masses and spins) of these simulations to be consistent with estimates of the parameters for GW150914 [1, 2]. Note that we are not presenting any additional information on parameter estimates in this paper. The parameters we chose are consistent with LIGO's observation of GW150914, but we could have chosen different parameters that are also consistent with the observation. As discussed in Ref. [1, 2], there is considerable uncertainty in parameter estimates for GW150914, particularly for the black-hole spins.

By comparing the results from these two codes, our study extends the statement made in the caption of Fig. 1 of Ref. [1]: that the numerical relativity calculations shown there are "confirmed to $99.9 \%$." Our investigation extends previous validation studies of each code internally [31, 32], and against one another [33], using common standards for waveform accuracy [23], to current versions of both numerical-relativity codes for the important case of modeling GW150914.

SpEC and LazEv are completely independent. They use different formulations of Einstein's equations, so they assume different decompositions of $G_{\mu \nu}=8 \pi T_{\mu \nu}$ into evolution equations and constraints, and they solve for different dynamical variables. They use different methods to choose coordinates and different methods of handling the spacetime singularities inside the black holes. They use different analytic and numerical methods for generating constraint-satisfying initial data, and they use different geometries for the numerical grid. They use different numerical methods for the spacetime evolution, for refining the numerical grid, and for extracting the gravitational waveforms from the evolved variables. They share no subroutines in common. But despite these vast differences, we show in this paper that the two codes produce the same physics. This is a very strong test of both codes and of the analytical and numerical methods underlying them.

Moreover, our comparison itself began independently. While we based both simulations on the same mass and spin parameter estimates, we only began discussing the comparison when lower resolutions had finished and higher resolutions were already under way. We made no special effort to tune the codes or the simulations to agree with each other. In this way, we have demon- strated the agreement of our codes under realistic working conditions, where multiple groups independently perform rapid follow up to a LIGO observation.

These results build confidence that both numerical methods produce consistent physics, which in turn builds confidence in current and future studies making use of targeted numerical-relativity simulations to follow up LIGO observations. Targeted numerical-relativity simulations already feature in recent studies directly comparing data for GW150914 to catalogs of numericalrelativity simulations [34, 35] and in recent studies injecting numerical-relativity waveforms into LIGO data for GW150914, to help assess systematic errors in approximate waveform models [2, 3, 36].

This paper is organized as follows. In Sec. II we briefly describe the formalism and implementation of SpEC, used by the Simulating eXtreme Spacetimes (SXS) collaboration to numerically evolve BBHs. In Sec. III we describe the different formalism and code implementation of LazEv, used by the Rochester Institute of Technology (RIT) group. Table I summarizes the independent methods these two codes employ to construct and evolve initial data for black holes. In Sec. IV, we directly compare the waveforms produced by each approach to one another. In Sec. $\mathrm{V}$ we conclude with a discussion of the significance of those comparisons and implications for future comparisons of observations with numericalrelativity calculations.

\section{SIMULATIONS USING PSEUDOSPECTRAL, EXCISION METHODS}

Simulations labeled SXS are carried out using the Spectral Einstein Code (SpEC) [65]. Given initial BBH parameters, a corresponding weighted superposition of two boosted, spinning Kerr-Schild black holes [66] is constructed, and then the constraints are solved [40, $46,67]$ by a pseudospectral method to yield quasiequilibrium $[42,66]$ initial data. Small adjustments in the initial orbital trajectory are made iteratively to produce initial data with low eccentricity [48, 68, 69].

The initial data are evolved using a first-order representation [70] of a generalized harmonic formulation [54, 55, 71] of Einstein's equations, and using damped harmonic gauge [59, 72, 73]. The equations are solved pseudospectrally on an adaptively-refined [74, 75] spatial grid that extends from pure-outflow excision boundaries just inside apparent horizons [59, 76-79] to an artificial outer boundary. Adaptive time-stepping automatically achieves time steps of approximately the Courant limit. On the Cal State Fullerton cluster, ORCA, the simulation achieved a typical evolution speed of $O(100 M) /$ day for the highest resolution (here we measure simulation time in units of $M$, the total mass of the binary). After the holes merge, all variables are automatically interpolated onto a new grid with a single excision boundary inside the common apparent horizon [76, 77], and the 


\begin{tabular}{|c|c|c|}
\hline & LazEv & SpEC \\
\hline \multicolumn{3}{|l|}{ Initial data } \\
\hline $\begin{array}{l}\text { Formulation of Einstein constraint } \\
\text { equations }\end{array}$ & $\begin{array}{l}\text { conformal method using Bowen-York } \\
\text { solutions [37-39] }\end{array}$ & conformal thin sandwich $[38,40]$ \\
\hline Singularity treatment & puncture data $[41]$ & $\begin{array}{l}\text { quasi-equilibrium black-hole } \\
\text { excision [42-44] }\end{array}$ \\
\hline Numerical method & pseudo-spectral [45] & pseudo-spectral [46] \\
\hline Achieving low orbital eccentricity & post-Newtonian inspiral [47] & iterative eccentricity removal $[48,49]$ \\
\hline \multicolumn{3}{|l|}{$\begin{array}{l}\text { Evolution } \\
\end{array}$} \\
\hline $\begin{array}{l}\text { Formulation of Einstein evolution } \\
\text { equations }\end{array}$ & BSSNOK [50-52] & $\begin{array}{l}\text { first-order generalized harmonic with } \\
\text { constraint damping }[11,53-55]\end{array}$ \\
\hline Gauge conditions & evolved lapse and shift [56-58] & damped harmonic [59] \\
\hline Singularity treatment & moving punctures $[12,13]$ & excision $[60]$ \\
\hline Outer boundary treatment & Sommerfeld & $\begin{array}{l}\text { minimally-reflective, } \\
\text { constraint-preserving }[53,61]\end{array}$ \\
\hline Discretization & high-order finite-differences $[62,63]$ & pseudo-spectral methods \\
\hline Mesh refinement & adaptive mesh refinement [64] & $\begin{array}{l}\text { domain decomposition with spectral } \\
\text { adaptive mesh refinement }[46,59]\end{array}$ \\
\hline
\end{tabular}

TABLE I. A comparison of the two independent numerical relativity codes described in the text. Each code uses different techniques to construct and evolve initial data for BBHs and to extract the emitted gravitational radiation. This table is based on Table I of Ref. [18].

evolution is continued. Constraint-preserving boundary conditions [61, 70, 80] are imposed on the outer boundary, and no boundary conditions are required or imposed on the excision boundaries.

We use a pseudospectral fast-flow algorithm [81] to find apparent horizons, and we compute spins on these apparent horizons using the approximate Killing vector formalism of Cook, Whiting, and Owen [82, 83].

Gravitational wave extraction is done by three independent methods: direct extraction of the NewmanPenrose quantity $\Psi_{4}$ at finite radius [48, 76, 84], extraction of the strain $h$ by matching to solutions of the Regge-Wheeler-Zerilli-Moncrief equations at finite radius [85, 86], and Cauchy-Characteristic Extraction [8791]. The latter method directly provides gravitational waveforms at future null infinity, while for the former two methods the waveforms are computed at a series of finite radii and then extrapolated to infinity [92]. Differences between the different methods, and differences in extrapolation algorithms, can be used to place error bounds on waveform extraction. These waveform extraction errors are important for the overall error budget of the simulations, and are typically on the order of, or slightly larger than, the numerical truncation error [93, 94]. In this paper, the waveforms we compare use Regge-WheelerZerilli-Moncrief extraction and extrapolation to infinity. We have verified that our choice of extrapolation order does not significantly affect our results. We have also checked that corrections to the wave modes [95] to account for a small drift in the coordinates of the center of mass have a negligible effect on our results.

\section{SIMULATIONS USING FINITE-DIFFERENCE, MOVING-PUNCTURE METHODS}

RIT simulations evolve $\mathrm{BBH}$ data sets using the LAzEV [63] implementation of the moving puncture approach $[12,13]$ with the conformal function $W=\sqrt{\chi}=$ $\exp (-2 \phi)$ suggested by Ref. [96]. For the simulation presented here, we use centered, sixth-order finite differencing in space [97], a fourth-order Runge-Kutta time integrator, ${ }^{1}$ and a 7 th-order Kreiss-Oliger dissipation operator. This sixth-order spatial finite difference scheme allows us to gain a factor $\sim 4 / 3$ in efficiency with the respect to the eighth-order implementation, because it reduces the number of ghost zones from 4 to 3 . We also allowed for a Courant factor $\mathrm{CFL}=1 / 3$ instead of the previous $\mathrm{CFL}=0.25$ [98] gaining another speedup factor of $4 / 3$. We verified that for this relaxing of the time integration step we still conserve the horizon masses and spins of the individual black holes during evolution and the phase of the gravitational waveforms to acceptable levels. This plus the use of the new XSEDE supercomputer Comet at SDSC ${ }^{2}$ lead to typical evolution speeds of $250 \mathrm{M} /$ day on 16 nodes for $N 100$ and similar for the higher resolution runs given the good weak scaling of LazEv. Note that our previous [99, 100] comparable simulations averaged $\sim 100 \mathrm{M} /$ day.

RIT's code uses the EInsteinToolkit [19, 101] / Cactus [102] / CARPet [64] infrastructure. The CARPET mesh refinement driver provides a "moving boxes" style of mesh refinement. In this approach, refined grids

\footnotetext{
1 Note that we do not upwind the advection terms.

2 https://portal.xsede.org/sdsc-comet
} 
of fixed size are arranged about the coordinate centers of both holes. The CARPET code then moves these fine grids about the computational domain by following the trajectories of the two black holes.

We use AHFinderDiREcT [103] to locate apparent horizons. We measure the magnitude of the horizon spin using the isolated horizon (IH) algorithm detailed in Ref. [104] and as implemented in Ref. [105]. Note that once we have the horizon spin, we can calculate the horizon mass via the Christodoulou formula

$$
m=\sqrt{m_{\mathrm{irr}}^{2}+S^{2} /\left(4 m_{\mathrm{irr}}^{2}\right)},
$$

where $m_{\text {irr }}=\sqrt{A /(16 \pi)}, A$ is the surface area of the apparent horizon, and $S$ is the spin angular momentum of the black hole (in units of $M^{2}$ ). In the tables below, we use the variation in the measured horizon irreducible mass and spin during the simulation as a measure of the error in computing these quantities. We measure radiated energy, linear momentum, and angular momentum, in terms of the radiative Weyl Scalar $\Psi_{4}$, using the formulas provided in Refs. [106, 107]. However, rather than using the full $\Psi_{4}$, we decompose it into $\ell$ and $m$ modes and solve for the radiated linear momentum, dropping terms with $\ell>6$. The formulas in Refs. [106, 107] are valid at $r=\infty$. We extract the radiated energy-momentum at finite radius and extrapolate to $r=\infty$. We find that the new perturbative extrapolation described in Ref. [108] provides the most accurate waveforms. While the difference of fitting both linear and quadratic extrapolations provides an independent measure of the error.

\section{RESULTS}

To model GW150914, we used each of the two codes to simulate a nonprecessing, unequal mass binary with the spin of the larger black hole aligned with the orbital angular momentum, and the spin of the smaller black hole antialigned. The initial data parameters for both codes are given in Tables II-III ${ }^{3}$. These tables show that the parameters of the two simulations are not exactly identical, primarily because we constructed and evolved the initial data independently but also because making the parameters exactly the same is challenging, given how different the methods for specifying initial data are in the two approaches. The largest difference is that the SXS simulation starts earlier in the inspiral than the RIT case, but also the two simulations have different orbital eccentricities and very small differences in the initial masses.

Both simulations start with a relatively small binary separation, so the entire evolution through coalescence

\footnotetext{
3 Note that data from the SXS configuration, including the gravitational waveform and the masses and spins as functions of time, are publicly available as simulation SXS:BBH:0305 at http://www.black-holes.org/waveforms
}

requires a time of roughly a few thousand $M$, where $M$ is the sum of the Christodoulou masses of both black holes. Because the mass ratio is near unity and the spins are small, this simulation is not difficult to perform for modern numerical relativity codes; for both codes considered here, this simulation requires about 7 to 10 days to complete. For each code, both simulations were repeated several times using different values of a parameter controlling the numerical resolution. Increasing the resolution results in higher accuracy, but requires more computation time and resources. Running multiple resolutions provides checks that the results converge with increasing resolution, and also provides an error estimate. The LazEv simulation was performed at three resolutions labeled $N 100, N 110$, and $N 120$, where $N 110$ and $N 120$ represent a global increase of the finite difference resolution by factors of 1.1 and 1.2, respectively, compared to the $N 100$ case. The SpEC simulation was performed at multiple resolutions labeled $L 0$ through $L 6$ in order of increasing resolution; $L 6$ represents a spectral adaptive-mesh-refinement error tolerance that is a factor of $e$ smaller than that of $L 5$.

The top panel of Fig. 1 displays the $(\ell, m)=(2,2)$ spin-weighted spherical harmonic mode of the gravitational waveform extracted from the two simulations, at the lowest resolution. The differences between these two simulations are not visible at this scale. Because of the finite signal-to-noise ratio of GW150914, the statistical error in the waveform reconstruction reported in Figs. 12 of Ref. [1] is far larger than the differences seen here. Note that we take a deliberately conservative approach to alignment here: we only apply a constant time shift (setting the peaks of the $(2,2)$ modes to $t=0$ ) and a constant phase shift (setting the phase to zero radians at $t=-0.6 \mathrm{~s}$. Later (Fig. 3 and Table IV), we will compare the mismatch of the waveforms, which (separately for each mode) optimizes over constant time and phase offsets and weights the difference inversely to LIGO's noise [Eq. (3)].

To better see the differences between the waveforms, the lower panel of Fig. 1 zooms in on the difference between the $(\ell, m)=(2,2)$ modes, and Fig. 2 plots fractional amplitude differences and phase differences, including not only the $(\ell, m)=(2,2)$ mode but several of the most significant higher modes. In Fig. 2, as in Fig. 1, we apply a constant time shift so that the peak $(\ell, m)=(2,2)$ occurs at time $t=0$ and a constant phase shift so each wave has a phase of zero radians at time $t=-0.6$ seconds. Differences between resolutions of each simulation estimate the numerical error. The differences between the SXS and RIT simulations' highest resolutions is far too small to be visible in a plot like the top panel of Fig. 1 or in Fig. 1 of Ref. [1], which compares the waveforms to LIGO's measured gravitational-wave strain for GW150914. Nevertheless, the differences are larger than our estimates of the numerical errors.

We suspect that the level of disagreement is determined in part by effects from small differences in the 


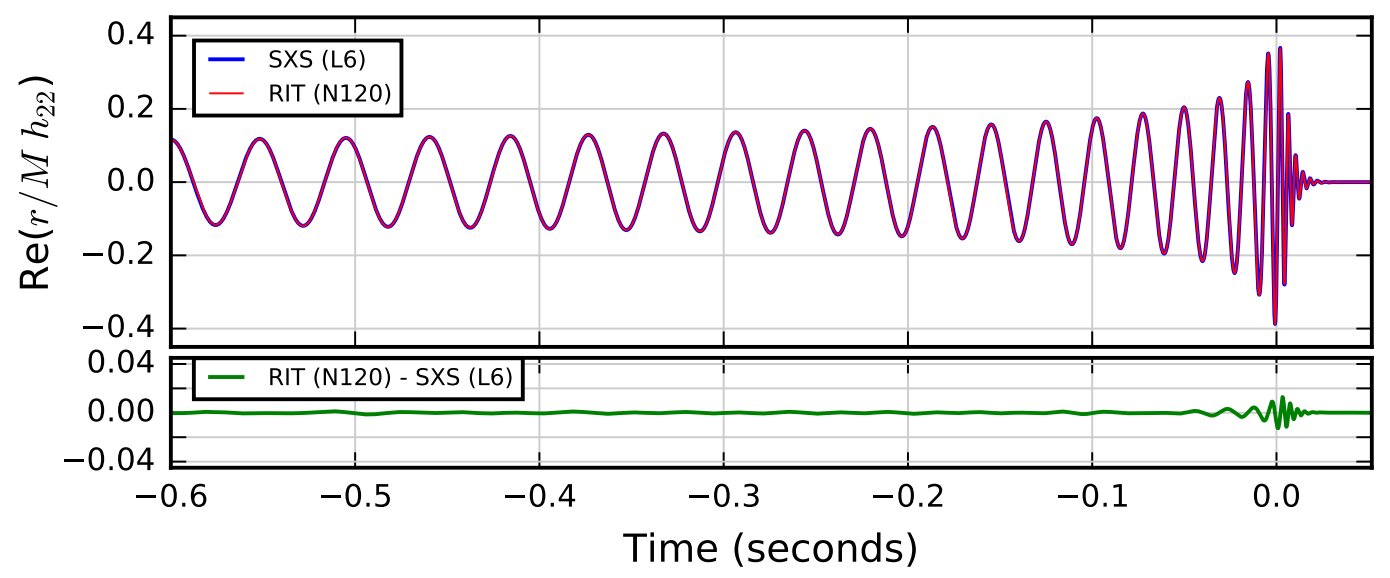

FIG. 1. A comparison of the $(\ell, m)=(2,2)$ mode extracted from the two fiducial simulations from SpEC and LazEv. Time is shown in seconds for a total mass of $70 M_{\odot}$. The bottom panel zooms in to show the difference between the SpEC and LazEv (2,2) modes, using resolutions are $N 120$ (LazEv) and L6 (SpEC). Here, we take a conservative approach to assessing differences in the waveforms: we apply a constant time shift to each waveform, so that the peak $(2,2)$ amplitude is at $t=0$, and a constant phase offset to each waveform, so that the phase is zero radians at $t=-0.6 \mathrm{~s}$. Table IV and Fig. 2 , in contrast, assess differences by computing the match, a comparison weighted by LIGO's noise. Note that lower resolution versions of these waveforms were used in the comparison in the caption of Fig. 1 of Ref. [1], which reported the agreement (match) of these waveforms as 99.9\%, using LIGO's noise curves for GW150914 [1].

\begin{tabular}{lccccccccccc}
\hline \hline Config. & $x_{1} / M$ & $x_{2} / M$ & $P_{t} / M$ & $P_{r} / M$ & $m_{1}^{p} / M$ & $m_{2}^{p} / M$ & $S_{1} / M^{2}$ & $S_{2} / M^{2}$ & $m_{1} / M$ & $m_{2} / M$ & $M_{\mathrm{ADM}} / M$ \\
\hline RIT & -6.7308 & 5.5192 & 0.083116 & -0.000490 & 0.40207 & 0.51363 & -0.08932 & 0.09963 & 0.45055 & 0.54945 & 0.99141 \\
SXS & -7.8597 & 6.4004 & - & - & - & - & -0.08918 & 0.09975 & 0.45020 & 0.54980 & 0.99235 \\
\hline \hline
\end{tabular}

TABLE II. Initial data parameters for the quasi-circular configurations with an spinning smaller mass black hole (labeled 1), and a larger mass spinning black hole (labeled 2). For the RIT configuration, the punctures are located at $\vec{r}_{1}=\left(x_{1}, 0,0\right)$ and $\vec{r}_{2}=\left(x_{2}, 0,0\right)$, with momenta $P= \pm\left(P_{r}, P_{t}, 0\right)$, spins $\vec{S}_{i}=\left(0,0, S_{i}\right)$, mass parameters $m^{p} / M$, horizon (Christodoulou) masses $m_{1} / M$ and $m_{2} / M$, total ADM mass $M_{\mathrm{ADM}}$, and spin angular momenta $S_{1} / M^{2}$ and $S_{2} / M^{2}$. For the SXS configuration, the apparent horizons begin with centers, in the asymptotically inertial coordinate frame, at coordinates $\left(x_{1}, y_{2}, 0\right)$ and $\left(x_{2}, y_{2}, 0\right)$, where $y_{1}=y_{2}=-0.0335 M$. In both cases, $M=m_{1}+m_{2}$ is the sum of the Christodoulou masses of the two black holes. The two codes specify initial data differently, so not all parameters are relevant for both codes.

initial configurations (mass, spin, eccentricity, etc.). Another potential source of systematic error is differences in wave extraction: LazEv computes $\Psi_{4}$ and then integrates to find the gravitational-wave strain $h$, while SpEC computed the waves shown in Fig. 2 by matching to Regge-Wheeler-Zerilli-Moncrief solutions. Differences between SpEC and LazEv also become large at late times in the ringdown, when the wave amplitude becomes smaller than the numerical error in the simulations.

To quantify the magnitude of the differences between these waveforms, we use the match,

$$
\mathscr{M} \equiv \frac{\left\langle h_{1} \mid h_{2}\right\rangle}{\sqrt{\left\langle h_{1} \mid h_{1}\right\rangle\left\langle h_{2} \mid h_{2}\right\rangle}}
$$

as implemented via a complex overlap as described in Eq. (2) in Ref. [109]:

$$
\left\langle h_{1} \mid h_{2}\right\rangle=2 \int_{-\infty}^{\infty} \frac{d f}{S_{n}(f)}\left[\tilde{h}_{1}(f) \tilde{h}_{2}(f)^{*}\right],
$$

where $\tilde{h}(f)$ is the Fourier transform of $h(t)$ and $S_{n}(f)$ is the power spectral density of the detector noise (here, taken to be the advanced LIGO design power spectrum [110]). When we compute the match using Eq. (3), we maximize (separately for each mode) over an overall constant time shift and an overall constant phase shift.

For our match calculations, we conservatively adopt the design-sensitivity noise power spectrum of advanced LIGO [111]; if we instead would compute matches using the observed $\mathrm{O} 1$ sensitivity, the matches would be larger. For instance, the $(\ell, m)=(2,2)$ mode would have a match of $99.9 \%$ (the basis of the "confirmed to 99.9\%" statement in Ref. [1]), instead of $99.8 \%$, as shown here. Table IV shows the match between the $(l, m)$ spin-weighted spherical harmonic gravitational waveform modes of different RIT resolutions versus the SXS L6 resolution, for a total mass of $70 M_{\odot}$. To account for finite simulation duration, we set the lower frequency of our match calculation to $m \times 11 \mathrm{~Hz}$. Note that for the $m=4$ and $m=5$ modes, which are much less significant than 


\begin{tabular}{lccccccc}
\hline \hline Config. & $q$ & $\chi_{1}$ & $\chi_{2}$ & $M \Omega_{0}$ & $M \dot{a}_{0} \times 10^{4}$ & $d_{0} / M$ & $e$ \\
\hline RIT & 1.220 & -0.4400 & 0.3300 & 0.02118 & -1.1712 & 12.2500 & 0.0012 \\
SXS & 1.221 & -0.4400 & 0.3300 & 0.01696 & -0.5306 & 14.2601 \\
\hline \hline
\end{tabular}

TABLE III. Physical properties of the initial black holes at time $t=0$. The table shows the mass ratio $q=m_{2} / m_{1}$ and dimensionless spins $\chi_{1}=S_{1} / m_{1}^{2}$ and $\chi_{2}=S_{2} / m_{2}^{2}$. The table also shows the initial orbital properties of both configurations. The holes begin at coordinate separation $d_{0}$ with initial orbital angular frequency $\Omega_{0}$ and initial radial expansion $\dot{a}_{0}$. Each hole's initial coordinate radial velocity $v_{r}$ and coordinate distance from the center of mass $r_{0}$ are related to the expansion by $\dot{a}_{0}=v_{r} / r_{0}$. These initial data result in an initial orbital eccentricity $e$. Here $M=m_{1}+m_{2}$ is the sum of the Christodoulou masses of the two black holes.
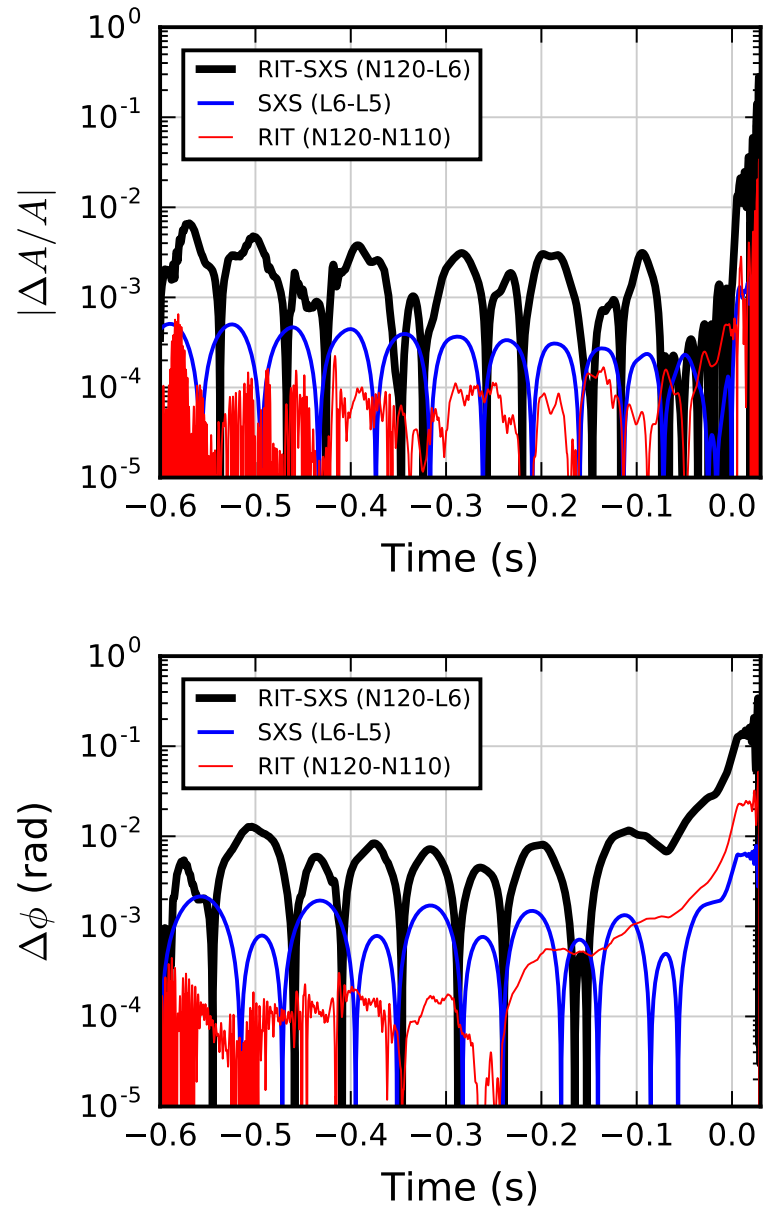

FIG. 2. Fractional differences in amplitude $\Delta A / A$ and differences in phase $\Delta \phi$ for the SXS and RIT simulations as a function of time (shown in seconds for a total mass of $70 M_{\odot}$ ) assuming zero inclination. We show numerical error estimates for the SXS and RIT simulations (obtained by subtracting different resolutions) and the difference between the highest resolution SXS and RIT simulations. Here, as in Fig. 1, we apply only an overall constant time shift and an overall constant phase shift to the waveforms, setting time $t=0$ at the peak of the $(\ell, m)=(2,2)$ modes and the phase to zero radians at $t=-0.6 \mathrm{~s}$. The differences include both the dominant $(\ell, m)=(2, \pm 2)$ mode and also the modes $(2, \pm 1),(3, \pm 3)$, $(3, \pm 2),(4, \pm 4)$, and $(4, \pm 3)$.

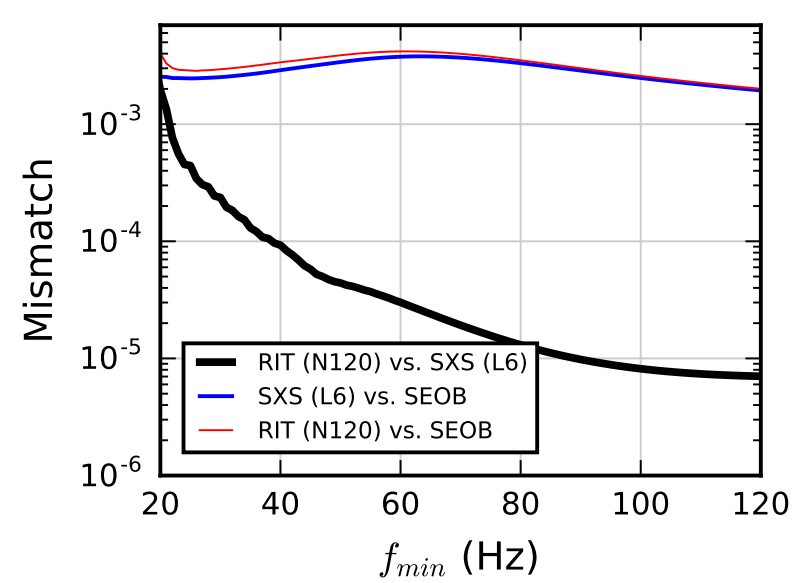

FIG. 3. Match between SXS and RIT full numerical $(2,2)$ mode waveforms, against one another and against a corresponding SEOBNRv2 template, for our fiducial parameters, scaled to a total detector frame binary system mass of $70 M_{\odot}$. For a sense of scale, at this mass the SXS and RIT waveforms start at $15.7 \mathrm{~Hz}$ and $19.5 \mathrm{~Hz}$, respectively. The SEOB waveform is evaluated starting at $10 \mathrm{~Hz}$. The match calculations adopt the Advanced LIGO design noise spectrum [111].

the dominant $(\ell, m)=(2,2)$ mode, this is above the frequency of $35 \mathrm{~Hz}$ where the GW150914 signal first entered the LIGO band. For each mode, the beginning of each waveform (a duration of several $M$ in time) is tapered, to reduce transient effects in their Fourier transforms. For the dominant $(2,2)$ mode, the match is close to unity for all RIT resolutions. Most other significant modes also display a high degree of agreement, particularly for $\ell \leq 3$. The table also shows the overlap of L6 with itself (with minimum frequency $22 \mathrm{~Hz}$ in all cases, to indicate the relative importance of each mode. Some modes that are much less significant than the $(2,2)$ mode have low matches but are (except for some of the least significant modes) convergent, suggesting that higher numerical resolution is necessary to accurately compute these high order modes.

To provide a sense of scale for this comparison, we also evaluate the match between the two simulations' $(\ell, m)=(2,2)$ modes and the same modes of a template waveform with the same parameters (i.e., the same 


\begin{tabular}{rccccr}
\hline \hline$\ell$ & $m$ & $N 100$ & $N 110$ & $N 120$ & $\left\langle h_{\ell m}^{L 6} \mid h_{\ell m}^{L 6}\right\rangle$ \\
\hline 2 & 0 & 0.8854 & 0.8863 & 0.8870 & 9.82 \\
2 & 1 & 0.9905 & 0.9914 & 0.9908 & 16.78 \\
2 & 2 & 0.9980 & 0.9980 & 0.9980 & 927.74 \\
\hline 3 & 0 & 0.7822 & 0.8146 & 0.8356 & 1.02 \\
3 & 1 & 0.9517 & 0.9569 & 0.9582 & 1.52 \\
3 & 2 & 0.9978 & 0.9980 & 0.9981 & 28.59 \\
3 & 3 & 0.9927 & 0.9933 & 0.9933 & 42.17 \\
\hline 4 & 0 & 0.3603 & 0.3581 & 0.3554 & 0.05 \\
4 & 1 & 0.7910 & 0.8348 & 0.8616 & 0.17 \\
4 & 2 & 0.9074 & 0.9425 & 0.9562 & 1.79 \\
4 & 3 & 0.9844 & 0.9909 & 0.9938 & 2.50 \\
4 & 4 & 0.9863 & 0.9886 & 0.9901 & 40.95 \\
\hline 5 & 0 & 0.3638 & 0.4050 & 0.4458 & 0.01 \\
5 & 1 & 0.2994 & 0.3652 & 0.4227 & 0.01 \\
5 & 2 & 0.6108 & 0.6176 & 0.6392 & 0.14 \\
5 & 3 & 0.7813 & 0.8709 & 0.9197 & 0.32 \\
5 & 4 & 0.9705 & 0.9815 & 0.9879 & 2.49 \\
5 & 5 & 0.9315 & 0.9552 & 0.9696 & 4.94 \\
\hline \hline
\end{tabular}

TABLE IV. Match between individual spherical harmonic modes $(\ell, m)$ of the SXS and RIT waveforms, using the advanced LIGO design sensitivity. The successively higher resolution simulations from RIT, labeled as N100, N110, N120 are compared to the L6 (highest) resolution run from SXS. The minimal frequency is taken as $f_{\min }=11 \mathrm{~m} \mathrm{~Hz}$ for $m>1$ and $f_{\min }=22 \mathrm{~Hz}$ for $m=0,1$ for a fiducial total mass of $M=70 M_{\odot}$. The column labeled $\left\langle h_{\ell m}^{L 6} \mid h_{\ell m}^{L 6}\right\rangle$ shows the overlap of L6 with itself, with a minimum frequency of $22 \mathrm{~Hz}$ in all cases, to indicate the significance of the mode.

mass ratio and spins) generated by SEOBNRv2 [112], one of the approximate, analytic waveform models used to infer GW150914's properties in Ref. [2]. Figure 3 shows the mismatch (1 minus the match), for successively higher starting cutoff frequencies, $f_{\min }$, above 20 $\mathrm{Hz}$. At high frequencies, the two simulations are much more consistent with one another than with the semianalytic SEOBNR model. Below about $20 \mathrm{~Hz}$, the two simulations disagree with each other and with SEOBNRv2, but this is because the RIT simulation starts at $19.5 \mathrm{~Hz}$ and the SXS simulation starts at $15.7 \mathrm{~Hz}$, and because the waveforms from both simulations are tapered. Note that in order to respond quickly to GW150914, to reduce computation time both of these simulations were chosen to start at higher frequencies than typical for full numerical simulations. Therefore, the low-frequency mismatch shown in Fig. 3 overstates the differences between the two codes for longer, more typical simulations.

The evolution of the aligned spinning binary leads to remnant masses, spins and recoil velocities as shown in Table V. They display an excellent agreement between the two codes, to at least three significant figures, and they appear convergent with increasing resolution. The fraction $E_{\mathrm{rad}} / M$ of the initial mass $M$ radiated as gravitational waves can be inferred (via energy conservation) to be

$$
\frac{E_{\mathrm{rad}}}{M}=1-\frac{m_{\mathrm{rem}}}{M}
$$

\begin{tabular}{cccc}
\hline \hline$\#$ & $m_{\text {rem }} / M$ & $\chi_{\text {rem }}^{z}$ & $V_{\text {rem }}^{x y}(\mathrm{~km} / \mathrm{s})$ \\
\hline N100 & 0.952015 & 0.691961 & 131.79 \\
N110 & 0.952020 & 0.691965 & 133.35 \\
N120 & 0.952021 & 0.691969 & 134.38 \\
L0 & 0.951760 & 0.691863 & 136.78 \\
L2 & 0.951971 & 0.692030 & 137.22 \\
L4 & 0.952000 & 0.692119 & 136.00 \\
L6 & 0.952033 & 0.692085 & 134.17 \\
\hline \hline
\end{tabular}

TABLE V. Remnant results for spinning binaries. We show the remnant mass $m_{r e m}$ in units of the total initial mass $M \equiv$ $m_{1}+m_{2}$, the remnant dimensionless spin $\chi_{r e m}^{z} \equiv J_{r e m}^{z} / m_{r e m}^{2}$, and the remnant velocity in the x-y plane $V_{r e m}^{x y}$. We show results for different LazEv resolutions (N100, N110, and N120) and different SpEC resolutions (L0, L2, L4, and L6).

The SXS and RIT simulations agree that $1-m_{\text {rem }} / M=$ $4.80 \%$ of the initial mass is radiated as gravitational waves. For GW150914, whose initial mass in the source frame is $65_{-4}^{+5} M_{\odot}$ [2], the radiated energy predicted by the SXS and RIT simulations, $3.1 M_{\odot}$, is consistent with the estimate of $3.0 \pm 0.5 M_{\odot}$ given in Ref. [1].

\section{DISCUSSION}

We have demonstrated that two completely independent codes to evolve binary black holes (SpEC and LazEv) produce very similar results. As shown in Fig. 1 and Table IV, we find good agreement even with moderately low resolution simulations (i.e. N100 and L5). A detailed convergence analysis, like that summarized in Table IV, suggests that both the generalized harmonic [11] and moving puncture [12, 13] approaches lead to accurate solutions of the general relativity field equations. Given that the initial configurations are not exactly the same (different eccentricities, slightly different masses and spins), we consider this general agreement an excellent verification of the analytic formulations, numerical methods, and code implementations used in both SpEC and LazEv.

The next steps in further verifying the results of numerical relativity codes will be to consider binary systems with precession and to consider simulations that follow a larger number of binary orbits. For these more demanding tests, it will be more important to start different codes with closely coordinated initial parameters. This study will be the subject of a future publication.

Future work also includes considering cases with more extreme parameters. Here, the simulations' very good agreement with the SEOB waveform is not surprising, since the moderate spins and almost equal masses make this an especially easy region of the parameter space to model. But for higher mass ratios and more extreme spins, numerical relativity might disagree more strongly with semianalytic, approximate waveforms, especially in regions where the semianalytic models have not been 
tuned to numerical relativity. Recent studies have begun exploring the agreement of numerical relativity and approximate, analytic waveforms in different regions of the BBH parameter space [21, 27, 35, 113, 114].

However, from the results of Fig. 3 we can already conclude that even if analytic waveform models provide a very good approximation to the true prediction of general relativity, full numerical solutions of Einstein's equations can be visibly more accurate than analytic models. Targeted followup with numerical relativity is thus an important tool for comparing gravitational-wave observation and theory and for reliably measuring potential deviations from Einstein's theory of gravitation [3].

Our study suggests that both groups' standard production simulations are sufficiently accurate and efficient to respond rapidly and comprehensively to further events like GW150914, informing the analysis and interpretation of LIGO data. Followup simulations of events like GW150914 can be performed on a timescale of days to weeks (depending on resolution) and at low computational cost, with confidence that both methods produce consistent physics. This is important for the construction of numerically generated waveform data banks with simulations from heterogeneous codes and formalisms.

However, numerical-relativity simulations can be considerably more costly and challenging elsewhere in the BBH parameter space, particularly if they remain in LIGO's band for more orbits, such as GW151226, or if they have more extreme parameters. For instance, the SpEC simulation modeling GW151226 that appears in Fig. 5 of Ref. [4] (SXS:BBH:0317 at http:// black-holes org/waveforms) required approximately 2 months to complete, and a recent simulation similar to those used here to model GW150914 but with spins $\chi=+0.96$ for the larger black hole and $\chi=-0.9$ for the smaller black hole (SXS:BBH:0306 at http:// black-holes.org/waveforms) required approximately two months to complete. Future work includes enabling more rapid, targeted follow up numerical-relativity simulations for these more challenging cases.

\section{ACKNOWLEDGMENTS}

We are pleased to acknowledge Joshua R. Smith and Jocelyn S. Read for helpful discussions of this work and Patricia Schmidt for helpful discussions and for helping us to implement the numerical-relativity injection HDF5 file format [115] she developed with Ian Harry, which facilitated some of the comparisons in this paper. We gratefully acknowledge the NSF for financial support from grant Nos. PHY-0969855, PHY1212426, PHY-1305730, PHY-1306125, PHY-1307489, PHY-1229173, PHY-1404569, PHY-1606522, PHY1607520, ACI-1550436, AST-1028087, AST-1333129, AST-1333520, OCI-0832606, and DRL-1136221. The authors are also grateful for support from the Sherman Fairchild Foundation, the NSERC of Canada, the Canada Research Chairs Program, and the Canadian Institute for Advanced Research. Computational resources were provided by the ORCA cluster at California State University, Fullerton (CSUF), supported by CSUF, NSF grant No. PHY-142987, and the Research Corporation for Science Advancement; by XSEDE allocations TG-PHY060027N and TG-PHY990007N; by NewHorizons and BlueSky Clusters at Rochester Institute of Technology, which were supported by NSF grant Nos. PHY-0722703, DMS-0820923, AST-1028087, and PHY-1229173; by the GPC supercomputer at the SciNet HPC Consortium [116], which is funded by the Canada Foundation for Innovation (CFI) under the auspices of Compute Canada, the Government of Ontario, the Ontario Research Fund (ORF) - Research Excellence, and the University of Toronto; and by the Zwicky cluster at Caltech, which is supported by the Sherman Fairchild Foundation and by NSF award PHY-0960291.
[1] B. P. Abbott et al. (Virgo, LIGO Scientific), Phys. Rev. Lett. 116, 061102 (2016), arXiv:1602.03837 [gr-qc].

[2] B. P. Abbott et al. (LIGO Scientific Collaboration, Virgo Collaboration), Phys. Rev. Lett. 116, 241102 (2016), arXiv:1602.03840 [gr-qc].

[3] B. P. Abbott et al. (LIGO Scientific Collaboration, Virgo Collaboration), Phys. Rev. Lett. 116, 221101 (2016), arXiv:1602.03841 [gr-qc].

[4] B. P. Abbott et al. (Virgo, LIGO Scientific), Phys. Rev. Lett. 116, 241103 (2016), arXiv:1606.04855 [gr-qc].

[5] B. P. Abbott et al. (the Virgo, The LIGO Scientific), (2016), arXiv:1606.04856 [gr-qc].

[6] B. Abbott et al., Astrophys. J. Lett. 818, 22 (2016), https://dcc.ligo.org/LIGO-P1500262/public/main.

[7] S. Hahn and R. Lindquist, Ann. Phys. (NY) 29, 304 (1964).

[8] L. Smarr, The Structure of General Relativity with a Numerical Illustration: The Collision of Two Black Holes,
Ph.D. thesis, University of Texas at Austin (1975).

[9] L. Smarr, in Eighth Texas Symposium on Relativistic Astrophysics, Annals of the New York Academy of Sciences, Vol. 302, edited by M. D. Papagiannis (1977) p. 569.

[10] A. M. Abrahams et al. (Binary Black Hole Grand Challenge Alliance), Phys. Rev. Lett. 80, 1812 (1998), grqc/9709082.

[11] F. Pretorius, Phys. Rev. Lett. 95, 121101 (2005), arXiv:gr-qc/0507014 [gr-qc].

[12] M. Campanelli, C. Lousto, P. Marronetti, and Y. Zlochower, Phys. Rev. Lett. 96, 111101 (2006), arXiv:grqc/0511048 [gr-qc].

[13] J. G. Baker, J. Centrella, D.-I. Choi, M. Koppitz, and J. van Meter, Phys. Rev. Lett. 96, 111102 (2006), arXiv:gr-qc/0511103 [gr-qc].

[14] M. W. Choptuik, L. Lehner, and F. Pretorius, in General Relativity and Gravitation, edited by A. Ashtekar, 
B. K. Berger, J. Isenberg, and M. MacCallum (Cambridge University Press, 2015) pp. 361-411, cambridge Books Online, arXiv:1502.06853 [gr-qc].

[15] U. Sperhake, General Relativity and Gravitation 46, 1 (2014).

[16] A. Le Tiec, Int. J. Mod. Phys. D 23, 1430022 (2014), arXiv:1408.5505 [gr-qc].

[17] M. Hannam, Gen. Rel. Grav. 46, 1767 (2014), arXiv:1312.3641 [gr-qc].

[18] H. P. Pfeiffer, Class. Quantum Grav. 29, 124004 (2012), arXiv:1203.5166 [gr-qc].

[19] F. Löffler, J. Faber, E. Bentivegna, T. Bode, P. Diener, et al., Class. Quantum Grav. 29, 115001 (2012), arXiv:1111.3344 [gr-qc].

[20] A. H. Mroue, M. A. Scheel, B. Szilagyi, H. P. Pfeiffer, M. Boyle, D. A. Hemberger, L. E. Kidder, G. Lovelace, S. Ossokine, N. W. Taylor, A. Zenginoglu, L. T. Buchman, T. Chu, E. Foley, M. Giesler, R. Owen, and S. A. Teukolsky, Phys. Rev. Lett. 111, 241104 (2013), arXiv:1304.6077 [gr-qc].

[21] B. Szilagyi, J. Blackman, A. Buonanno, A. Taracchini, H. P. Pfeiffer, et al., Phys. Rev. Lett. 115, 031102 (2015), arXiv:1502.04953 [gr-qc].

[22] P. Ajith, M. Boyle, D. A. Brown, B. Brugmann, L. T. Buchman, et al., Class. Quantum Grav. 29, 124001 (2012).

[23] I. Hinder et al. (The NRAR Collaboration), Class. Quantum Grav. 31, 025012 (2014), arXiv:1307.5307 [grqc].

[24] L. Pekowsky, R. O'Shaughnessy, J. Healy, and D. Shoemaker, Phys. Rev. D 88, 024040 (2013), arXiv:1304.3176 [gr-qc].

[25] J. Healy, C. O. Lousto, and Y. Zlochower, Phys. Rev. D 89, 104052 (2014), arXiv:1406.7295 [gr-qc].

[26] J. Clark, L. Cadonati, J. Healy, I. Heng, J. Logue, N. Mangini, L. London, L. Pekowsky, and D. Shoemaker, in Gravitational Wave Astrophysics, Astrophysics and Space Science Proceedings, Vol. 40, edited by C. F. Sopuerta (Springer International Publishing, Cham, Switzerland, 2015) pp. 281-287, arXiv:1406.5426 [gr-qc].

[27] S. Husa, S. Khan, M. Hannam, M. Prrer, F. Ohme, X. Jiménez Forteza, and A. Bohé, Phys. Rev. D93, 044006 (2016), arXiv:1508.07250 [gr-qc].

[28] B. Aylott, J. G. Baker, W. D. Boggs, M. Boyle, P. R. Brady, et al., Class. Quantum Grav. 26, 165008 (2009), arXiv:0901.4399 [gr-qc].

[29] B. Aylott et al., Class. Quantum Grav. 26, 114008 (2009), arXiv:0905.4227 [gr-qc].

[30] J. Aasi et al. (The LIGO Scientific Collaboration, the Virgo Collaboration, the NINJA-2 Collaboration), Class. Quantum Grav. 31, 115004 (2014), arXiv:1401.0939 [gr-qc].

[31] J. G. Baker, M. Campanelli, F. Pretorius, and Y. Zlochower, Class. Quantum Grav. 24, S25 (2007), grqc/0701016.

[32] M. Hannam, S. Husa, J. G. Baker, M. Boyle, B. Bruegmann, T. Chu, N. Dorband, F. Herrmann, I. Hinder, B. J. Kelly, L. E. Kidder, P. Laguna, K. D. Matthews, J. R. van Meter, H. P. Pfeiffer, D. Pollney, C. Reisswig, M. A. Scheel, and D. Shoemaker, Phys. Rev. D 79, 084025 (2009), arXiv:0901.2437 [gr-qc].

[33] P. Ajith, M. Boyle, D. A. Brown, B. Brügmann, L. T. Buchman, L. Cadonati, M. Campanelli, T. Chu, Z. B.
Etienne, S. Fairhurst, M. Hannam, J. Healy, I. Hinder, S. Husa, L. E. Kidder, B. Krishnan, P. Laguna, Y. T. Liu, L. London, C. O. Lousto, G. Lovelace, I. MacDonald, P. Marronetti, S. Mohapatra, P. Mösta, D. Müller, B. C. Mundim, H. Nakano, F. Ohme, V. Paschalidis, L. Pekowsky, D. Pollney, H. P. Pfeiffer, M. Ponce, M. Pürrer, G. Reifenberger, C. Reisswig, L. Santamaría, M. A. Scheel, S. L. Shapiro, D. Shoemaker, C. F. Sopuerta, U. Sperhake, B. Szilágyi, N. W. Taylor, W. Tichy, P. Tsatsin, and Y. Zlochower, Classical and Quantum Gravity 29, 124001 (2012), arXiv:1201.5319 [gr-qc].

[34] B. P. Abbott et al. (Virgo, LIGO Scientific), (2016), arXiv:1606.01262 [gr-qc].

[35] K. Jani, J. Healy, J. A. Clark, L. London, P. Laguna, and D. Shoemaker, (2016), arXiv:1605.03204 [gr-qc].

[36] B. P. Abbott et al. (Virgo, LIGO Scientific), (2016), arXiv:1606.01210 [gr-qc].

[37] N. Ó. Murchadha and J. W. York, Jr., Phys. Rev. D 10, 428 (1974).

[38] H. P. Pfeiffer and J. W. York, Phys. Rev. D 67, 044022 (2003).

[39] J. M. Bowen and J. W. York, Jr., Phys. Rev. D 21, 2047 (1980).

[40] J. W. York, Phys. Rev. Lett. 82, 1350 (1999).

[41] S. Brandt and B. Brügmann, Phys. Rev. Lett. 78, 3606 (1997).

[42] M. Caudill, G. B. Cook, J. D. Grigsby, and H. P. Pfeiffer, Phys. Rev. D 74, 064011 (2006), gr-qc/0605053.

[43] G. B. Cook and H. P. Pfeiffer, Phys. Rev. D 70, 104016 (2004).

[44] G. B. Cook, Phys. Rev. D 65, 084003 (2002).

[45] M. Ansorg, B. Brügmann, and W. Tichy, Phys. Rev. D 70, 064011 (2004), gr-qc/0404056.

[46] H. P. Pfeiffer, L. E. Kidder, M. A. Scheel, and S. A. Teukolsky, Comput. Phys. Commun. 152, 253 (2003), gr-qc/0202096.

[47] S. Husa, M. Hannam, J. A. González, U. Sperhake, and B. Brügmann, Phys. Rev. D 77, 044037 (2008), arXiv:0706.0904 [gr-qc].

[48] H. P. Pfeiffer, D. A. Brown, L. E. Kidder, L. Lindblom, G. Lovelace, and M. A. Scheel, Class. Quantum Grav. 24, S59 (2007), gr-qc/0702106.

[49] A. Buonanno, L. E. Kidder, A. H. Mroué, H. P. Pfeiffer, and A. Taracchini, Phys. Rev. D 83, 104034 (2011).

[50] T. Nakamura, K. Oohara, and Y. Kojima, Prog. Theor. Phys. 90 (1987).

[51] M. Shibata and T. Nakamura, Phys. Rev. D 52, 5428 (1995).

[52] T. W. Baumgarte and S. L. Shapiro, Phys. Rev. D 59, 024007 (1999), gr-qc/9810065.

[53] L. Lindblom, M. A. Scheel, L. E. Kidder, R. Owen, and O. Rinne, Class. Quantum Grav. 23, S447 (2006), arXiv:gr-qc/0512093v3 [gr-qc].

[54] H. Friedrich, Commun. Math. Phys. 100, 525 (1985).

[55] F. Pretorius, Class. Quantum Grav. 22, 425 (2005).

[56] C. Bona, J. Massó, E. Seidel, and J. Stela, Phys. Rev. D 56, 3405 (1997).

[57] M. Alcubierre, B. Brügmann, P. Diener, M. Koppitz, D. Pollney, E. Seidel, and R. Takahashi, Phys. Rev. D 67, 084023 (2003).

[58] J. R. van Meter, J. G. Baker, M. Koppitz, and D.-I. Choi, Phys. Rev. D 73, 124011 (2006), gr-qc/0605030.

[59] B. Szilágyi, L. Lindblom, and M. A. Scheel, Phys. Rev. 
D 80, 124010 (2009), arXiv:0909.3557 gr-qc].

[60] M. A. Scheel, H. P. Pfeiffer, L. Lindblom, L. E. Kidder, O. Rinne, and S. A. Teukolsky, Phys. Rev. D 74, 104006 (2006).

[61] O. Rinne, L. Lindblom, and M. A. Scheel, Class. Quantum Grav. 24, 4053 (2007).

[62] S. Husa, J. A. González, M. Hannam, B. Brügmann, and U. Sperhake, Class. Quantum Grav. 25, 105006 (2008).

[63] Y. Zlochower, J. Baker, M. Campanelli, and C. Lousto, Phys. Rev. D 72, 024021 (2005), arXiv:gr-qc/0505055 [gr-qc].

[64] E. Schnetter, S. H. Hawley, and I. Hawke, Class. Quantum Grav. 21, 1465 (2004).

[65] http://www.black-holes.org/SpEC.html.

[66] G. Lovelace, R. Owen, H. P. Pfeiffer, and T. Chu, Phys. Rev. D 78, 084017 (2008).

[67] S. Ossokine, F. Foucart, H. P. Pfeiffer, M. Boyle, and B. Szilágyi, (2015), arXiv:1506.01689, arXiv:1506.01689 [gr-qc].

[68] A. Buonanno, L. E. Kidder, A. H. Mroué, H. P. Pfeiffer, and A. Taracchini, Phys. Rev. D 83, 104034 (2011), arXiv:1012.1549 [gr-qc].

[69] A. H. Mroué and H. P. Pfeiffer, (2012), arXiv:1210.2958 [gr-qc].

[70] L. Lindblom, M. A. Scheel, L. E. Kidder, R. Owen, and O. Rinne, Class. Quantum Grav. 23, S447 (2006), grqc/0512093.

[71] D. Garfinkle, Phys. Rev. D 65, 044029 (2002).

[72] L. Lindblom and B. Szilágyi, Phys. Rev. D 80, 084019 (2009), arXiv:0904.4873.

[73] M. W. Choptuik and F. Pretorius, Phys. Rev. Lett. 104, 111101 (2010), arXiv:0908.1780 [gr-qc].

[74] G. Lovelace, M. A. Scheel, and B. Szilágyi, Phys. Rev. D 83, 024010 (2011), arXiv:1010.2777 [gr-qc].

[75] B. Szilágyi, Int. J. Mod. Phys. D 23, 1430014 (2014), arXiv:1405.3693 [gr-qc].

[76] M. A. Scheel, M. Boyle, T. Chu, L. E. Kidder, K. D. Matthews and H. P. Pfeiffer, Phys. Rev. D 79, 024003 (2009), arXiv:gr-qc/0810.1767.

[77] D. A. Hemberger, M. A. Scheel, L. E. Kidder, B. Szilágyi, G. Lovelace, N. W. Taylor, and S. A. Teukolsky, Class. Quantum Grav. 30, 115001 (2013), arXiv:1211.6079 [gr-qc].

[78] S. Ossokine, L. E. Kidder, and H. P. Pfeiffer, Phys. Rev. D 88, 084031 (2013), arXiv:1304.3067 [gr-qc].

[79] M. A. Scheel, M. Giesler, D. A. Hemberger, G. Lovelace, K. Kuper, M. Boyle, B. Szilágyi, and L. E. Kidder, Class. Quantum Grav. 32, 105009 (2015), arXiv:1412.1803 [gr-qc].

[80] O. Rinne, Class. Quantum Grav. 23, 6275 (2006).

[81] C. Gundlach, Phys. Rev. D 57, 863 (1998).

[82] G. B. Cook and B. F. Whiting, Phys. Rev. D 76, 041501(R) (2007).

[83] R. Owen, Topics in Numerical Relativity: The periodic standing-wave approximation, the stability of constraints in free evolution, and the spin of dynamical black holes, Ph.D. thesis, California Institute of Technology (2007).

[84] M. Boyle, D. A. Brown, L. E. Kidder, A. H. Mroué, H. P. Pfeiffer, M. A. Scheel, G. B. Cook, and S. A. Teukolsky, Phys. Rev. D 76, 124038 (2007), arXiv:0710.0158 [grqc].

[85] L. T. Buchman and O. C. A. Sarbach, Class. Quantum
Grav. 24, S307 (2007).

[86] O. Rinne, L. T. Buchman, M. A. Scheel, and H. P. Pfeiffer, Class. Quantum Grav. 26, 075009 (2009).

[87] N. T. Bishop, R. Gomez, L. Lehner, M. Maharaj, and J. Winicour, Phys. Rev. D56, 6298 (1997), arXiv:grqc/9708065.

[88] J. Winicour, Living Rev. Rel. 8 (2005).

[89] R. Gomez, W. Barreto, and S. Frittelli, Phys. Rev. D 76, 124029 (2007), arXiv:0711.0564 [gr-qc].

[90] C. Reisswig, N. T. Bishop, and D. Pollney, Gen. Rel. Grav. 45 (5), 1069 (2013), arXiv:1208.3891 [gr-qc].

[91] C. J. Handmer and B. Szilágyi, Class. Quantum Grav. 32, 025008 (2015), arXiv:1406.7029.

[92] M. Boyle and A. H. Mroué, Phys. Rev. D 80, 124045 (2009), arXiv:0905.3177 [gr-qc].

[93] N. W. Taylor, M. Boyle, C. Reisswig, M. A. Scheel, T. Chu, L. E. Kidder, and B. Szilágyi, Phys. Rev. D 88, 124010 (2013), arXiv:1309.3605 [gr-qc].

[94] T. Chu, H. Fong, P. Kumar, H. P. Pfeiffer, M. Boyle, D. A. Hemberger, L. E. Kidder, M. A. Scheel, and B. Szilagyi, (2015), arXiv:1512.06800 [gr-qc].

[95] M. Boyle, Phys. Rev. D 93, 084031 (2016).

[96] P. Marronetti, W. Tichy, B. Bruegmann, J. Gonzalez, and U. Sperhake, Phys. Rev. D 77, 064010 (2008), arXiv:0709.2160 [gr-qc].

[97] C. O. Lousto and Y. Zlochower, Phys. Rev. D 77, 024034 (2008), arXiv:0711.1165 [gr-qc].

[98] Y. Zlochower, M. Ponce, and C. O. Lousto, Phys. Rev. D 86, 104056 (2012), arXiv:1208.5494 [gr-qc].

[99] C. O. Lousto and Y. Zlochower, Phys. Rev. D 88, 024001 (2013), arXiv:1304.3937 [gr-qc].

[100] C. O. Lousto and J. Healy, Phys. Rev. D 93, 044031 (2016), arXiv:1506.04768 [gr-qc].

[101] Einstein Toolkit home page: http://einsteintoolkit.org.

[102] "The Cactus Computational Toolkit," http://www. cactuscode.org.

[103] J. Thornburg, Class. Quantum Grav. 21, 743 (2004), gr-qc/0306056.

[104] O. Dreyer, B. Krishnan, D. Shoemaker, and E. Schnetter, Phys. Rev. D 67, 024018 (2003).

[105] M. Campanelli, C. O. Lousto, Y. Zlochower, B. Krishnan, and D. Merritt, Phys. Rev. D 75, 064030 (2007), arXiv:gr-qc/0612076 [gr-qc].

[106] M. Campanelli and C. O. Lousto, Phys. Rev. D 59, 124022 (1999), arXiv:gr-qc/9811019 [gr-qc].

[107] C. O. Lousto and Y. Zlochower, Phys. Rev. D 76, 041502 (2007), arXiv:gr-qc/0703061 [GR-QC].

[108] H. Nakano, J. Healy, C. O. Lousto, and Y. Zlochower, Phys. Rev. D 91, 104022 (2015), arXiv:1503.00718 [grqc].

[109] H.-S. Cho, E. Ochsner, R. O'Shaughnessy, C. Kim, and C.-H. Lee, Phys. Rev. D87, 024004 (2013), arXiv:1209.4494 [gr-qc].

[110] LIGO Scientific Collaboration, "Advanced ligo anticipated sensitivity curves," (2011), https://dcc.ligo. org/LIGO-T0900288/public.

[111] LIGO Scientific Collaboration, Virgo Collaboration, B. P. Abbott, R. Abbott, T. D. Abbott, M. Abernathy, T. Accadia, F. Acernese, and et al., Living Reviews in Relativity 19 (2016), 10.1007/lrr-2016-1, arXiv:1304.0670 [gr-qc].

[112] A. Taracchini, A. Buonanno, Y. Pan, T. Hinderer, M. Boyle, D. A. Hemberger, L. E. Kidder, G. Lovelace, 
A. H. Mroue, H. P. Pfeiffer, M. A. Scheel, B. Szilagyi, N. W. Taylor, and A. Zenginoglu, Phys. Rev. D 89 (R), 061502 (2014), arXiv:1311.2544 [gr-qc].

[113] P. Kumar, T. Chu, H. Fong, H. P. Pfeiffer, M. Boyle, D. A. Hemberger, L. E. Kidder, M. A. Scheel, and B. Szilagyi, Phys. Rev. D93, 104050 (2016), arXiv:1601.05396 [gr-qc].

[114] P. Kumar, K. Barkett, S. Bhagwat, N. Afshari, D. A. Brown, G. Lovelace, M. A. Scheel, and B. Szilgyi, Phys. Rev. D 92, 102001 (2015), arXiv:1507.00103 [gr-qc].
[115] P. Schmidt, I. Harry, and H. Pfeiffer, Numerical Relativity Injection Infrastructure, Tech. Rep. LIGOT1500606-v2 (LIGO Scientific Collaboration report) https://dcc.ligo.org/LIGO-T1500606-v2/public.

[116] C. Loken, D. Gruner, L. Groer, R. Peltier, N. Bunn, M. Craig, T. Henriques, J. Dempsey, C.-H. Yu, J. Chen, L. J. Dursi, J. Chong, S. Northrup, J. Pinto, N. Knecht, and R. V. Zon, J. Phys.: Conf. Ser. 256, 012026 (2010). 